\title{
STABILIZER RIGIDITY IN IRREDUCIBLE GROUP ACTIONS
}

\author{
YAIR HARTMAN AND OMER TAMUZ
}

\begin{abstract}
We consider irreducible actions of locally compact product groups, and of higher rank semi-simple Lie groups. Using the intermediate factor theorems of Bader-Shalom and NevoZimmer, we show that the action stabilizers, and all irreducible invariant random subgroups, are co-amenable in their normal closure. As a consequence, we derive rigidity results on irreducible actions that generalize and strengthen the results of Bader-Shalom and Stuck-Zimmer.
\end{abstract}

\section{Contents}

1. Introduction

1.1. Applications

1.2. Related work

1.3. Acknowledgments

2. The Chabauty topology and the normal closure of an IRS

3. Poisson bundles and co-amenable IRSs

3.1. Random walks on groups and the Furstenberg-Poisson boundary

3.2. Coset spaces

3.3. Poisson bundles

3.4. Co-amenable IRSs

4. Intermediate factor theorems

4.1. Poisson bundles as intermediate factors

4.2. IFT actions and co-amenable IRSs

5. Proofs of main theorems and corollaries

References

Date: October 25, 2016.

Y. Hartman is supported by the European Research Council, grant 239885. O. Tamuz is supported by ISF grant 1300/08, and is a recipient of the Google Europe Fellowship in Social Computing. This research is supported in part by this Google Fellowship. 


\section{INTRODUCTION}

Let $G$ be a locally compact second countable (lcsc) group. An invariant random subgroup (IRS) of $G$ is a random variable that takes values in $\mathrm{Sub}_{G}$, the space of closed subgroups of $G$, and whose distribution is invariant to conjugation by any element of $G[2$. IRSs arise naturally as stabilizers of probability measure preserving (pmp) actions, and in fact any IRS is the stabilizer of some pmp action (see [1, Theorem 2.4] and also [2,7]). They are also an interesting object of study as stochastic generalizations of normal subgroups, and of lattices.

Let $G=G_{1} \times G_{2}$ be a product of two lcsc groups. A pmp action $G \curvearrowright(X, m)$ is irreducible (with respect to the decomposition $G=$ $\left.G_{1} \times G_{2}\right)$ if the actions of both $G_{1}$ and $G_{2}$ are ergodic. Likewise, a pmp action of a semi-simple Lie group is said to be irreducible if the action of every non-central closed normal subgroup is ergodic. An IRS $K$ in $G$ is irreducible if $G \curvearrowright\left(\mathrm{Sub}_{G}, \lambda\right)$ is irreducible, where $\lambda$ is the distribution of $K$ and $G$ acts on $\operatorname{Sub}_{G}$ by conjugation [1.

In this paper we study irreducible IRSs of product groups and of semi-simple Lie groups. Our results are are generalizations of the theorems of Bader-Shalom [3, Theorem 1.6] (for product groups) and of Stuck-Zimmer [23] (for semi-simple Lie groups), who both require $G$ to have property $(\mathrm{T})$; we explore what can be said when this hypothesis is removed. Nevertheless, in both cases, we rely on the corresponding Intermediate Factor Theorems: Bader-Shalom [3] and and NevoZimmer [20].

To state our results we will need to recall the following definition: A subgroup $H$ is said to be co-amenable in $G$ if there exists a $G$-invariant mean on $G / H$ [8, 19, 22]; equivalently, one can define co-amenability as a fixed point property or as a representation theoretical property, in analogy to the different equivalent definitions of amenability (see [8] or [22, Theorem 4.18]). A normal subgroup $N \triangleleft G$ is co-amenable in $G$ if and only if $G / N$ is amenable.

We say that an IRS $K$ is co-amenable in $G$ if it is almost surely coamenable in $G$. Likewise, if $K$ almost surely has some property (e.g., trivial, normal, co-finite), we say succinctly that $K$ has this property.

Theorem 1. Let $G=G_{1} \times G_{2}$ be a locally compact second countable group, and let $K$ be an irreducible IRS in $G$. Then there exist closed normal subgroups $N_{1} \triangleleft G_{1}$ and $N_{2} \triangleleft G_{2}$ such that $K$ is co-amenable in $N_{1} \times N_{2}$.

Theorem 2. Let $G$ be a connected semi-simple Lie group with finite center, no compact factors and $\mathbb{R}$-rank $\geq 2$. Let $K$ be an irreducible 
IRS in $G$. Then $K$ is either equal to a closed normal subgroup, or else $K$ is co-amenable in $G$.

As these theorems do not require the groups to have property (T), they provide rigidity results on the irreducible IRSs of groups such as $S L_{2}(\mathbb{R}) \times S L_{2}(\mathbb{R})$, to which the theorems of Bader-Shalom and StuckZimmer do not apply. We thus give a partial answer to a question asked in Stuck-Zimmer [23, page 731]. It remains unknown, however, whether $S L_{2}(\mathbb{R}) \times S L_{2}(\mathbb{R})$ has any irreducible co-amenable IRSs that are not, in fact, co-finite.

One can interpret Theorem 1 as addressing the following question: What IRSs does a product group admit? The irreducibility assumption rules outs the trivial example of a product of IRSs of each group. Thus, Theorem 1 says that, in a sense, there is not much else. A motivation for this question is the following stronger, basic statement that holds for normal subgroups:

Fact 1.1. Let $G=G_{1} \times G_{2}$ be a topological group. Given a closed normal subgroup $N \triangleleft G$, let $N_{1} \triangleleft G_{1}$ and $N_{2} \triangleleft G_{2}$ be the closures of the projections of $N$ to $G_{1}$ and $G_{2}$. Then $N$ is co-abelian in $N_{1} \times N_{2}$.

Our approach to these question involves the analysis of the FurstenbergPoisson boundary of the random walk on $G$ and on coset spaces of $G$. Our main technical contribution is in proving the following claim, which is a generalization to IRSs of an analogous claim that holds for normal subgroups, but not in general for non-normal subgroups.

Theorem 3. Let $G$ be a locally compact second countable group and let $\mu$ be a probability measure on $G$ that is equivalent to the Haar measure. Let $K \leq G$ be an IRS. If the Furstenberg-Poisson boundary of the $\mu$ random walk on $K \backslash G$ is almost surely trivial, then $K$ is co-amenable in $G$.

We in fact prove below a more general result (Theorem 3.3) which implies Theorem 3 .

1.1. Applications. The conclusions of Theorems 1 and 2 can be strengthened when more constraints are imposed on $G$. In particular, we consider the following notion: An lcsc group is said to be just non-amenable if every closed normal subgroup is co-amenable. Note that if a group is simple or just non-compact then it is also just non-amenable.

Corollary 1.2. Let $G=G_{1} \times G_{2}$ be a locally compact second countable group, and let both $G_{1}$ and $G_{2}$ be just non-amenable. Then every irreducible IRS is either co-amenable in $G$ or equal to a normal subgroup. 
As noted above, this holds in particular when $G_{1}$ and $G_{2}$ are simple. In that case, it is tempting to conjecture (see Stuck-Zimmer [23, page 731]) that in fact every irreducible IRS is either equal to a normal subgroup or is co-finite; recall that $K \leq G$ is said to be co-finite if there exists a $G$-invariant finite measure on $G / K 1$. Bader and Shalom [3] prove that this is indeed the case when $G_{1}$ and $G_{2}$ both have property (T). Their work continues the work of Stuck and Zimmer [23, who draw the same conclusions for high rank semi-simple Lie groups whose every simple factor has property $(\mathrm{T})$.

In the following two corollaries we show that it suffices that only one of the factors have property $(\mathrm{T})$, both in the product group setting and in the Lie group setting.

Corollary 1.3. Let $G=G_{1} \times G_{2}$ be a locally compact second countable group, and let $G_{1}$ be just non-compact and have property (T). Let $G \curvearrowright$ $(X, m)$ be a faithful irreducible pmp action.

Then the action $G \curvearrowright(X, m)$ is either essentially free or essentially transitive. It follows that the associated stabilizer IRS is either trivial or co-finite in $G$.

This constitutes a strengthening of the Essentially Free Actions Theorem of Bader-Shalom; they require that both $G_{1}$ and $G_{2}$ have property (T) and be just non-compact.

Corollary 1.4. Let $G$ be a connected semi-simple Lie group with finite center, no compact factors and $\mathbb{R}$-rank $\geq 2$. Assume that one of the simple factors of $G$ has property $(T)$. Then any faithful irreducible pmp G-action is either essentially free or essentially transitive, and its associated stabilizer IRS is either trivial or a lattice in $G$.

Finally, we show that similar results can be derived without property $(\mathrm{T})$, given that $G_{1}$ is simple non-amenable and $G_{2}$ is simple discrete.

Corollary 1.5. Let $G_{1}$ be a simple, non-amenable, locally compact second countable group, and let $G_{2}$ be a simple, countable, discrete group. Then every non-trivial irreducible pmp action of $G_{1} \times G_{2}$ is essentially free. It follows that every irreducible IRS in $G$ is equal to a normal subgroup.

1.2. Related work. Recently, Creutz [6] proved Corollary [1.4 independently, using a different approach. In the same paper he also generalizes Bader-Shalom's theorem to the case that $G_{1}$ has property (T) and both $G_{1}$ and $G_{2}$ are simple.

\footnotetext{
${ }^{1}$ In fact, co-finite IRSs admit some more structure: any ergodic co-finite IRS is supported on a single orbit $\left\{H^{g}\right\}_{g \in G}$, for some co-finite $H \leq G$ (see Corollary 5.6). In other words, the $G$-action on the IRS is essentially transitive.
} 
Creutz and Peterson [7] prove similar rigidity results for irreducible lattices and commensurators of lattices in semi-simple Lie groups, and also for product groups with the Howe-Moore property and property (T).

In [1] it is shown that in the setting of Corollary 1.4, if $G$ has property (T) then every irreducible IRS is either equal to a normal subgroup or is a lattice.

1.3. Acknowledgments. We would like to thank Uri Bader, Amos Nevo, Jesse Peterson and Benjamin Weiss for useful discussions and motivating conversations. We would also like to thank Yehuda Shalom and Lewis Bowen for helpful comments on an early draft of this article.

\section{The Chabauty topology and the normal Closure of an IRS}

Let $X$ be a locally compact topological space. The space of all closed subsets of $X, \mathcal{C}(X)$, admits a natural topology called the compact topology, under which it is a compact Hausdorff space (see, e.g. [25]). If $G$ is a locally compact group, then $\operatorname{Sub}_{G} \subset \mathcal{C}(G)$, the set of closed subgroups of $G$, is a closed subset and the induced topology on $\operatorname{Sub}_{G}$ is known as the Chabauty topology [5]. If $G$ is furthermore second countable then $\mathrm{Sub}_{G}$ is a metrizable space, and in particular is second countable.

In the Chabauty topology, a sequence $\left\{H_{n}\right\}$ of subgroups in $\mathrm{Sub}_{G}$ converges to $H \in \mathrm{Sub}_{G}$ if and only if

(1) For every $h \in H$ there exists a sequence $h_{n} \rightarrow h$ such that $h_{n} \in H_{n}$.

(2) If $h_{n} \rightarrow h$ and $h_{n} \in H_{n}$ then $h \in H$.

$G$ acts naturally on $\mathrm{Sub}_{G}$ by conjugation, and under the Chabauty topology this action is continuous. An invariant random subgroup (IRS) is a Borel probability measure on $\mathrm{Sub}_{G}$ which is invariant under this conjugation action. Note that this is a slight (but standard) abuse of nomenclature; more precisely, an IRS is a random variable $K$ taking values in $\mathrm{Sub}_{G}$ whose law is a conjugation invariant Borel probability measure. We denote the space of all IRSs of a given group $G$ by $\operatorname{IRS}(G)$. The term invariant random subgroup was introduced by Abért, Glasner, and Virág [1, although the mathematical object has been studied earlier - for example by Stuck and Zimmer [23].

Given an IRS, we want to define its normal closure, which is the smallest subgroup on which the IRS "lives"; an IRS $K$ lives in some $H<G$ if $K$ is almost surely contained in $H$. 
Definition 2.1. Let $K$ be an IRS in $G$ with law $\lambda$. The normal closure of $\lambda$, denoted $\overline{\langle\lambda\rangle}$, is the minimal closed subgroup in $G$ that almost surely contains $K$ :

$$
\overline{\langle\lambda\rangle}=\min \left\{H \in \operatorname{Sub}_{G}: \lambda\left(\left\{H^{\prime}: H^{\prime} \leq H\right\}\right)=1\right\} .
$$

Equivalently, $\overline{\langle\lambda\rangle}=\min \left\{H \in \operatorname{Sub}_{G}: \lambda\left(\operatorname{Sub}_{H}\right)=1\right\}$, where $\operatorname{Sub}_{H}$ is the space of closed subgroups of $H$.

It is not obvious from this definition that the normal closure exists. However, provided that it exists, it is immediate that it is unique, and applying the conjugation invariance of $\lambda$ yields that it is normal.

The existence of the normal closure is established in the next proposition, which also provides an equivalent definition for it. Before stating the theorem we will introduce the following notation: For a Borel set $A \subseteq \mathrm{Sub}_{G}$ we denote by $\langle A\rangle$ the subgroup of $G$ generated by all the

elements of the all groups in $A$, and by $\overline{\langle A\rangle} \in \mathrm{Sub}_{G}$ the topological closure of this subgroup.

Proposition 2.2. Let $G$ be an lcsc group and let $\lambda \in \operatorname{IRS}(G)$. Then $\overline{\langle\lambda\rangle}=\overline{\langle\operatorname{supp} \lambda\rangle}$ : the normal closure of $\lambda$ is equal to the closure of the group generated by all the groups in the support of $\lambda$.

Proof. Let $K$ have distribution $\lambda$, and denote $N=\overline{\langle\lambda\rangle}$. Since $\operatorname{Sub}_{G}$ is second countable, $\operatorname{supp} \lambda$ is a $\lambda$-full measure set and so $K$ is almost surely a subgroup of $N$. It follows that $N \leq \overline{\langle\operatorname{supp} \lambda\rangle}$, by the definition of the normal closure.

Thus to prove the claim it suffices to show that that if $H \in \mathrm{Sub}_{G}$ is such that $K$ is almost surely a subgroup of $H$, then $\overline{\langle\operatorname{supp} \lambda\rangle}$ is a subgroup of $H$. Note that

(1) $\lambda\left(\mathrm{Sub}_{H}\right)=1$, since $K$ is almost surely a subgroup of $H$.

(2) $\mathrm{Sub}_{H}$ is a closed set in $\mathrm{Sub}_{G}$.

So $\mathrm{Sub}_{H}$ is a closed $\lambda$-full measure set, and as such must include $\operatorname{supp} \lambda$; here we again use the fact that $\mathrm{Sub}_{G}$ is second countable. It follows that $H$ includes $\overline{\langle\operatorname{supp} \lambda\rangle}$, and we have proved the claim.

\section{Poisson BUndles And CO-Amenable IRSs}

3.1. Random walks on groups and the Furstenberg-Poisson boundary. Let $G$ be a locally compact second countable group, and let $\mu$ be a probability measure on $G$ that is equivalent to the Haar 
measure; that is, let $\mu$ and the Haar measure be mutually absolutely continuous2. We will tersely say that $\mu$ is Haar-equivalent.

A $\mu$-random walk on a group $G$ is a measure $\mathbb{P}_{\mu}$ on $G^{\mathbb{N}}$ given by the push-forward of the product measure $\mu^{\mathbb{N}}$ under the map $\left(h_{1}, h_{2}, h_{3}, \ldots\right) \mapsto$ $\left(h_{1}, h_{1} h_{2}, h_{1} h_{2} h_{3}, \ldots\right)$. Equivalently, let $\left\{h_{n}\right\}_{n \in \mathbb{N}}$ be i.i.d. random variables with measure $\mu$, and let $g_{n}=h_{1} \cdots h_{n}$. Then a $\mu$-random walk is the distribution of $\left(h_{1}, h_{1} h_{2}, h_{1} h_{2} h_{3}, \ldots\right)=\left(g_{1}, g_{2}, \ldots\right)$.

The shift-action on $G^{\mathbb{N}}$ is given by $\left(g_{1}, g_{2}, g_{3}, \ldots\right) \mapsto\left(g_{2}, g_{3}, \ldots\right)$. The Furstenberg-Poisson boundary [9, 10] of a $\mu$-random walk, denoted by $\Pi(G, \mu)$, is Mackey's point realization [18] of the shift-invariant sigmaalgebra of $\left(G^{\mathbb{N}}, \mathbb{P}_{\mu}\right)$ (see, e.g., [4, 26]).

A related process is the $\mu$-random walk on coset spaces of $G$. Indeed, for any $H \in \operatorname{Sub}_{G}$, let $\left\{g_{n}\right\}_{n \in \mathbb{N}}$ be as above. Then $\left(H g_{1}, H g_{1} g_{2}, \ldots\right)$ is a $\mu$-random walk on $H \backslash G$.

3.2. Coset spaces. Let $\operatorname{Cos}_{G} \subset \mathcal{C}(G)$ denote the space of all left cosets of closed subgroups of $G$ :

$$
\operatorname{Cos}_{G}=\left\{g H: g \in G, H \in \operatorname{Sub}_{G}\right\} .
$$

As a closed subset in $\mathcal{C}(G)$, it is naturally equipped with the corresponding induced topology, and with a continuous $G$ left action given by $k(g H)=k g H$.

An equivalent definition is to let $\operatorname{Cos}_{G}$ be the space of right cosets

$$
\operatorname{Cos}_{G}=\left\{H g: g \in G, H \in \operatorname{Sub}_{G}\right\} .
$$

This is indeed equivalent since every left coset $g H=g H^{-1} g=H^{g} g$ is also a right coset. The description of $\operatorname{Cos}_{G}$ as a space of right cosets makes it clear that $G$ also acts on $\operatorname{Cos}_{G}$ from the right by $(H g) k=H g k$. Note that both the right and left actions on $\mathrm{Cos}_{G}$ are continuous, and that these two actions commute.

There are two natural projections $\pi_{l}, \pi_{r}: \operatorname{Cos}_{G} \rightarrow \mathrm{Sub}_{G}:$

$$
\begin{aligned}
\pi_{l}: g H & \mapsto H \\
\pi_{r}: H g & \mapsto H
\end{aligned}
$$

Note that $\pi_{r}$ is $G$-equivariant with respect to the left $G$-action on $\operatorname{Cos}_{G}$ and $\mathrm{Sub}_{G}$ :

$$
\left(\pi_{r}(H g)\right)^{k}=H^{k}=\pi_{r}\left(H^{k} k g\right)=\pi_{r}(k H g)
$$

\footnotetext{
${ }^{2}$ Some of our intermediate results could be generalized to more general $\mu$ (e.g., any $\mu$ absolutely continuous with respect to the Haar measure), but we choose not to pursue this, in order to simplify the proofs.
} 
and that it is invariant to the right $G$-action:

$$
\pi_{r}(H g)=H=\pi_{r}(H g k) .
$$

Similar statements can be made for $\pi_{l}$.

3.3. Poisson bundles. Kaimanovich introduces Poisson boundles in [14]. Later, these were also studied by Bowen in [4]. Fix $\lambda \in \operatorname{IRS}(G)$, let $\mu$ be a probability measure on $G$, and consider the space $\operatorname{Cos}_{G}^{\mathbb{N}}$, endowed with the product topology. $G$ acts on the left by the diagonal action.

A natural stochastic process on $\operatorname{Cos}_{G}^{\mathbb{N}}$ can be constructed as follows. Choose $K$ at random from $\lambda$, choose $\left(h_{1}, h_{1} h_{2}, \ldots\right)$ from $\mathbb{P}_{\mu}$, the $\mu$ random walk on $G$, and let $C_{n} \in \operatorname{Cos}_{G}$ be given by $K h_{1} h_{2} \cdots h_{n}$. Then $C_{n}$ has distribution $\lambda * \mu^{n}$ (here and below $*$ denotes convolution, and $\mu^{n}$ are the convolution powers of $\mu$ ), and it is easy to verify that $\left(C_{1}, C_{2}, \ldots\right)$ is a Markov chain on $\operatorname{Cos}_{G}^{\mathbb{N}}$ with initial distribution $\lambda * \mu$.

To define the measure of this Markov chain formally, equip $\operatorname{Cos}_{G}^{\mathbb{N}}$ with the measure $\mathbb{P}_{\mu}^{\lambda}$ given by the push-forward $\kappa_{*}\left(\mathbb{P}_{\mu} \times \lambda\right)$ where

$$
\begin{aligned}
\kappa: \quad G^{\mathbb{N}} \times \operatorname{Sub}_{G} & \longrightarrow \operatorname{Cos}_{G}^{\mathbb{N}} \\
\left(\left(g_{1}, g_{2}, \ldots\right), H\right) & \longmapsto\left(H g_{1}, H g_{2}, \ldots\right)
\end{aligned}
$$

Then the process $\left(C_{1}, C_{2}, \ldots\right)$ has distribution $\mathbb{P}_{\mu}^{\lambda}$.

It is straightforward to check that $\kappa$ is a $G$-equivariant map from $G^{\mathbb{N}} \times \operatorname{Sub}_{G}$ to $\operatorname{Cos}_{G}^{\mathbb{N}}$, and that $\mathbb{P}_{\mu}^{\lambda}$ is supported on elements of the form $\left(H g_{1}, H g_{2}, \ldots\right)$. On these, the left $G$-action on $\operatorname{Cos}_{G}^{\mathbb{N}}$ is given by

$$
\begin{aligned}
g\left(H g_{1}, H g_{2}, \ldots\right) & =\left(g H g_{1}, g H g_{2}, \ldots\right) \\
& =\left(H^{g} g g_{1}, H^{g} g g_{2}, \ldots\right) .
\end{aligned}
$$

Hence the measure $g_{*} \mathbb{P}_{\mu}^{\lambda}$ corresponds to choosing $H$ from $\lambda$ and then choosing a $\mu$-random walk on $H^{g} \backslash G$, starting at $H^{g} g$. Note, however, that since $\lambda$ is conjugation invariant, $H^{g}=g H^{-1}$ and $H$ have the same distribution. Note also that since $\mu$ is equivalent to the Haar measure then $\mathbb{P}_{\mu}^{\lambda}$ is G-quasi-invariant; in fact,

$$
\frac{d g_{*}\left(\mathbb{P}_{\mu} \times \lambda\right)}{d\left(\mathbb{P}_{\mu} \times \lambda\right)}\left(\left(g_{1}, g_{2}, \ldots\right), H\right)=\frac{d g_{*} \mu}{d \mu}\left(g_{1}\right),
$$

by the Markov property of the $\mu$-random walk on $G$. Hence $\mathbb{P}_{\mu} \times \lambda$ is $G$-quasi-invariant, and so its push-forward $\mathbb{P}_{\mu}^{\lambda}$ is likewise $G$-quasiinvariant.

Note that $\kappa$ is shift-equivariant, if we act in the obvious way by shifts on $G^{\mathbb{N}} \times \operatorname{Sub}_{G}$ and $\operatorname{Cos}_{G}^{\mathbb{N}}$. The shift-invariant sigma-algebra defines the Poisson bundle $\mathrm{B}_{\mu}(\lambda)$ : 
Definition 3.1. Given $\lambda \in \operatorname{IRS}(G)$, denote by $\mathrm{B}_{\mu}(\lambda)$ Mackey's point realization of the shift-invariant sigma-algebra on $\left(\operatorname{Cos}_{G}^{\mathbb{N}}, \mathbb{P}_{\mu}^{\lambda}\right)$. We shall refer to $\mathrm{B}_{\mu}(\lambda)$ as a Bowen space.

This presentation is slightly different than Bowen's [4], but only semantically so: Bowen defines $\mathrm{B}_{\mu}(\lambda)$ as a fiber bundle over $\left(\operatorname{Sub}_{G}, \lambda\right)$, where the fiber over $H<G$ is the Furstenberg-Poisson boundary of the $\mu$-random walk on $H \backslash G$. This is simply the disintegration of $\mathrm{B}_{\mu}(\lambda)$ with respect to the factor $\hat{\pi}_{r}: \mathrm{B}_{\mu}(\lambda) \rightarrow\left(\mathrm{Sub}_{G}, \lambda\right)$ defined in Proposition 4.3, see also (4.3) and the preceding paragraph. We encourage the reader to study the details in Bowens paper [4. For further discussion and another application of Poisson bundles see [11.

Another point of view is that, as the Mackey realization of the shiftinvariant sigma-algebra, the Poisson bundle $\mathrm{B}_{\mu}(\lambda)$ is the FurstenbergPoisson boundary of the Markov chain $\left(C_{1}, C_{2}, \ldots\right)$ described in the beginning of this section; this is simply the definition of the FurstenbergPoisson boundary of a Markov chain. Since $G$ acts on $\operatorname{Cos}_{G}^{\mathbb{N}}$, Mackey's realization provides us also with a $G$-action on $\mathrm{B}_{\mu}(\lambda)$. This action can be interpreted as follows: The application of $g \in G$ to $\mathrm{B}_{\mu}(\lambda)$, the Furstenberg-Poisson boundary of the Markov chain with initial distribution $\lambda * \mu$, yields the Furstenberg-Poisson boundary of the same Markov chain, with initial distribution $g_{*}(\lambda * \mu)=\lambda *\left(g_{*} \mu\right)$.

3.4. Co-amenable IRSs. The following result is due to Kaimanovich [13].

Theorem 3.2. Let $G$ be an lcsc group, and let $\mu$ be a Haar-Equivalent probability measure on $G$. Let $N^{\prime} \leq N$ be two closed normal subgroups of $G$, and let $\bar{\mu}$ be the projection of $\mu$ to $G / N^{\prime}$. If the $N$-action on the Furstenberg-Poisson boundary $\Pi\left(G / N^{\prime}, \bar{\mu}\right)$ is measure preserving, then $N^{\prime}$ is co-amenable in $N$.

In particular, if the Furstenberg-Poisson boundary of the $\bar{\mu}$-random walk on $G / N^{\prime}$ is trivial, then $N^{\prime}$ is co-amenable in $G$.

This theorem does not hold in general for non-normal subgroups. In this section we prove the following theorem, which shows that it does hold for IRSs.

Theorem 3.3 (Co-amenable IRSs). Let $G$ be an lcsc group, and let $\mu$ be a Haar-Equivalent probability measure on $G$. Let $K \leq G$ be an IRS with distribution $\lambda$, and let $N \triangleleft G$ be a closed normal group such that almost surely $K \leq N$. If the $N$-action on the Poisson bundle $\mathrm{B}_{\mu}(\lambda)$ is measure preserving, then $K$ is co-amenable in $N$.

In particular, if the Furstenberg-Poisson boundary of the $\mu$-random walk on $K \backslash G$ is almost surely trivial, then $K$ is co-amenable in $G$. 
The rest of this section is devoted to proving this theorem.

The next lemma can be deduced from known general results about the Furstenberg-Poisson boundaries of Markov chains (see, e.g., [12, Lemma 2.1]). We provide its proof here for the reader's convenience. Recall that we assume throughout that $\mu$ is equivalent to the Haar measure. We denote by $\|\cdot\|$ the total variation norm.

Lemma 3.4. Let $(B, \nu)=\mathrm{B}_{\mu}(\lambda)$. If $g_{*} \nu=\nu$ for some $g \in G$ then

$$
\lim _{n}\left\|g_{*} \lambda * \mu^{n}-\lambda * \mu^{n}\right\|=0 .
$$

Recall that $\lambda * \mu^{n}$ is the projection of $\mathbb{P}_{\mu}^{\lambda}$ on the $n^{\text {th }}$ coordinate, or the position of the random walk at time $n$. Likewise, $g_{*} \lambda * \mu^{n}=\lambda * g_{*} \mu^{n}$ is the position at time $n$ when the initial distribution of the random walk is $\lambda * g_{*} \mu$ rather than $\lambda * \mu$.

Intuitively, the Furstenberg-Poisson boundary distribution is the distribution of the random walk at time infinity. The theorem hypothesis $g_{*} \nu=\nu$ means that the Furstenberg-Poisson boundary is unchanged when the random walk is initially displaced by $g$. The claim is that under the same displacement, the distributions of the positions at large times $n$ are also similar.

Proof. Let $\mathcal{T}_{n}^{m}$ be the sigma-algebra of $\left(\operatorname{Cos}_{G}^{\mathbb{N}}, \mathbb{P}_{\mu}^{\lambda}\right)$ consisting of the events measurable in coordinates $(n, n+1, \ldots, m)$, and let $\mathcal{T}=\cap_{n} \mathcal{T}_{n}^{\infty}$ be the tail sigma-algebra. We first note that $\mathcal{T}$ and the shift-invariant sigma-algebra coincide, $\bmod \mathbb{P}_{\mu}^{\lambda}$ null sets. This is not true for general Markov chains, but it does hold for random walks on groups (see, e.g., [15]), from which it easily follows that it also holds here.

Hence $\mathrm{B}_{\mu}(\lambda)$ can be taken to be the Mackey point realization of $\mathcal{T}$, the tail sigma-algebra on $\left(\operatorname{Cos}_{G}^{\mathbb{N}}, \mathbb{P}_{\mu}^{\lambda}\right)$. Since $g_{*} \mathbb{P}_{\mu}^{\lambda}$ and $\mathbb{P}_{\mu}^{\lambda}$ are equivalent (see (3.1) and the subsequent paragraph), the hypothesis $g_{*} \nu=\nu$ means that

$$
\mathbb{P}_{\mu}^{\lambda}\left(g^{-1} T\right)=\mathbb{P}_{\mu}^{\lambda}(T) \text { for every tail event } T \in \mathcal{T} .
$$

Let

$$
d_{n}=\sup _{T \in \mathcal{T}_{n}^{\infty}}\left\|g_{*} \mathbb{P}_{\mu}^{\lambda}(T)-\mathbb{P}_{\mu}^{\lambda}(T)\right\|
$$

be the total variation distance between $g_{*} \mathbb{P}_{\mu}^{\lambda}(T)$ and $\mathbb{P}_{\mu}^{\lambda}(T)$, taken as measures over $\left(\operatorname{Cos}_{G}^{\mathbb{N}}, \mathcal{T}_{n}^{\infty}\right)$. Then by (say) the Martingale convergence theorem,

$$
\lim _{n} d_{n}=\sup _{T \in \mathcal{T}}\left\|g_{*} \mathbb{P}_{\mu}^{\lambda}(T)-\mathbb{P}_{\mu}^{\lambda}(T)\right\|=0
$$

where the second equality follows from (3.2). 
By the Markov property of this $\mu$-random walk, one can take the supremum in the definition of $d_{n}$ to be only over events measurable in the $n^{\text {th }}$ coordinate only:

$$
d_{n}=\sup _{T \in \mathcal{T}_{n}^{n}}\left\|g_{*} \mathbb{P}_{\mu}^{\lambda}(T)-\mathbb{P}_{\mu}^{\lambda}(T)\right\| .
$$

This expression is in turn equal to

$$
\frac{1}{2}\left\|g_{*} \lambda * \mu^{n}-\lambda * \mu^{n}\right\|
$$

since the projection of $\mathbb{P}_{\mu}^{\lambda}$ on its $n^{\text {th }}$ coordinate is $\lambda * \mu^{n}$. Thus

$$
\lim _{n}\left\|g_{*} \lambda * \mu^{n}-\lambda * \mu^{n}\right\|=2 \lim _{n} d_{n}=0 .
$$

A sequence of probability measures $\left\{\zeta_{n}\right\}$ on a $G$-space is almostinvariant if

$$
\lim _{n}\left\|g_{*} \zeta_{n}-\zeta_{n}\right\|=0
$$

for all $g \in G$.

Corollary 3.5. In the setting of Theorem 3.3, there exist $N$-almost invariant probability measures $\left\{\zeta_{n}\right\}$ on $\operatorname{Cos}_{G}$ such that $\pi_{l *}\left(g_{*} \zeta_{n}\right)=$ $\pi_{r *}\left(g_{*} \zeta_{n}\right)=\lambda$ for all $n \in \mathbb{N}$ and $g \in G$.

Proof. Let $\zeta_{n}=\lambda * \mu^{n}$, and let $(B, \nu)=\mathrm{B}_{\mu}(\lambda)$. Since $\nu$ is invariant to the $N$-action, it follows from Lemma 3.4 that for every $h \in N$

$$
\lim _{n}\left\|h_{*} \zeta_{n}-\zeta_{n}\right\|=0
$$

or, in other words, that the sequence $\left\{\zeta_{n}\right\}$ is $N$-almost invariant.

Recall that $\pi_{r}(H g)=H$ and $\pi_{l}(g H)=H$ for every $g H, H g \in \operatorname{Cos}_{G}$. Since

$$
\pi_{r *}\left(g_{*} \zeta_{n}\right)=\pi_{r *}\left(\lambda *\left(g_{*} \mu^{n}\right)\right)=\lambda,
$$

we have that $\pi_{r *} \zeta_{n}=\lambda$. And since $\lambda$ is conjugation invariant, we have that $\lambda *\left(g_{*} \mu^{n}\right)=\left(g_{*} \mu^{n}\right) * \lambda$, from which it follows that $\pi_{l *} \zeta_{n}=\lambda$.

In order to prove that $\lambda$ is co-amenable in $N$, we will demonstrate the existence of $N$-almost invariant measures on $N / H$, for $\lambda$-almost every $H$. These will be constructed as disintegrations of the $N$-almost invariant measures $\left\{\zeta_{n}\right\}$ on $\operatorname{Cos}_{G}$ from Corollary 3.5. Our first task will be to push the measures $\left\{\zeta_{n}\right\}$ forward to $N$-almost invariant measures on $\operatorname{Cos}_{N}$.

To this end, let rep: $G / N \rightarrow G$ be a measurable map which satisfies $\operatorname{rep}(g N) \in g N$; this is a section of the quotient map $g \mapsto g N$, or a map that chooses a representative for each coset of $N$ in $G$ in a measurable 
fashion. The existence of such a map was shown by Mackey [17, Lemma 1.1]. Let $\rho: G \rightarrow N$ be given by

$$
\rho(g)=g \cdot \operatorname{rep}\left(g^{-1} N\right) .
$$

Intuitively, $\rho(g)$ is the "difference" between $g$ and the representative of its $N$-coset. Note that $\rho$ is measurable and equivariant to the left $N$-action: for all $g \in G$ and $k \in N$ it holds that $\rho(k g)=k \rho(g)$.

Let $\operatorname{Cos}_{G, N}$ be the restriction of $\operatorname{Cos}_{G}$ to $G$-cosets of subgroups of $N$ :

$$
\operatorname{Cos}_{G, N}=\left\{H g \in \operatorname{Cos}_{G}: H \in \operatorname{Sub}_{N}\right\} .
$$

This closed subspace is equipped with a left $N$-action: for $k \in N$ and $H g \in \operatorname{Cos}_{G, N}, k(H g)=H^{k} k g \in \operatorname{Cos}_{G, N}$. This is simply the restriction of the $G$-action on $\operatorname{Cos}_{G}$.

For $H g \in \operatorname{Cos}_{G, N}$ it follows from the $N$-equivariance of $\rho$ that $\rho(H g)=H \rho(g) \in \operatorname{Cos}_{N}$. Hence we can extend $\rho$ to a map $\rho: \operatorname{Cos}_{G, N} \rightarrow$ $\operatorname{Cos}_{N}$. It is easy to check that this map too is equivariant with respect to the left $N$-action.

Now, $\pi_{r *}\left(g_{*} \zeta_{n}\right)=\lambda$ by Corollary 3.5 , and so our measures $\zeta_{n}$ are supported on $\operatorname{Cos}_{G, N}$. We can thus use $\rho$ to push-forward our $N$-almost invariant measures $\zeta_{n}$ on $\operatorname{Cos}_{G, N}$ to $N$-almost invariant measures on $\operatorname{Cos}_{N}: \alpha_{n}=\rho_{*} \zeta_{n}$. By again invoking the $N$-equivariance of $\rho$, it also follows that the projections $\pi_{r *} \alpha_{n}$ and $\pi_{l *} \alpha_{n}$ are equal to $\lambda$ (now as a measure on $\left.\operatorname{Sub}_{N}\right)$. We have thus proved the following claim.

Claim 3.6. There exist $N$-almost invariant probability measures $\alpha_{n}$ on $\operatorname{Cos}_{N}$ such that $\pi_{l *}\left(h_{*} \alpha_{n}\right)=\pi_{r *}\left(h_{*} \alpha_{n}\right)=\lambda$ for all $n \in \mathbb{N}$ and $h \in N$.

We are now ready to take the last step in the proof of Theorem 4.5.

Claim 3.7. $\lambda$ is co-amenable in $N$.

Proof. Let $\alpha_{n}$ be a sequence of probability measures on $\operatorname{Cos}_{N}$ given by Claim 3.6. Recall that $\pi_{l}: \operatorname{Cos}_{N} \rightarrow \operatorname{Sub}_{N}$ is given by $\pi_{l}(t H)=H$, and that $\pi_{l *}\left(t_{*} \alpha_{n}\right)=\lambda$ for all $t \in N$. We fix $t \in N$, and disintegrate both $\alpha_{n}$ and $t_{*} \alpha_{n}$ with respect to $\pi_{l}$ :

$$
\begin{aligned}
\alpha_{n} & =\int_{\operatorname{Sub}_{N}} \alpha_{n}^{H} \mathrm{~d} \lambda(H) \\
t_{*} \alpha_{n} & =\int_{\operatorname{Sub}_{N}}\left(t_{*} \alpha_{n}\right)^{H} \mathrm{~d} \lambda(H),
\end{aligned}
$$

so that $\alpha_{n}^{H}$ and $\left(t_{*} \alpha_{n}\right)^{H}$ are measures on $N / H$. Note that for every $k, k^{\prime} \in N$ it holds that $\pi_{l}\left(k k^{\prime} H\right)=\pi_{l}\left(k^{\prime} H\right)=H$. Hence both $\alpha_{n}^{H}$ and 
$t_{*}\left(\alpha_{n}^{H}\right)$ are supported on the same fiber (namely $\left.N / H\right)$, and we get that $\left(t_{*} \alpha_{n}\right)^{H}=t_{*}\left(\alpha_{n}^{H}\right)$.

As both $t_{*} \alpha_{n}$ and $\alpha_{n}$ are projected by $\pi_{l}$ to $\lambda$, we can disintegrate $t_{*} \alpha_{n}-\alpha_{n}$ to get

$$
\begin{aligned}
t_{*} \alpha_{n}-\alpha_{n} & =\int_{\operatorname{Sub}_{N}}\left(\left(t_{*} \alpha_{n}\right)^{H}-\alpha_{n}^{H}\right) \mathrm{d} \lambda(H) \\
& =\int_{\operatorname{Sub}_{N}}\left(t_{*}\left(\alpha_{n}^{H}\right)-\alpha_{n}^{H}\right) \mathrm{d} \lambda(H),
\end{aligned}
$$

and in particular,

$$
\left\|t_{*} \alpha_{n}-\alpha_{n}\right\|=\int_{\operatorname{Sub}_{G}}\left\|t_{*}\left(\alpha_{n}^{H}\right)-\alpha_{n}^{H}\right\| \mathrm{d} \lambda(H) .
$$

So for any $t \in N$ we have that $\left\|t_{*} \alpha_{n}-\alpha_{n}\right\| \rightarrow 0$, and therefore $\left\|t_{*}\left(\alpha_{n}^{H}\right)-\alpha_{n}^{H}\right\| \rightarrow 0$ for $\lambda$-almost every $H$. Finally, the existence of asymptotically invariant measures on $N / H$ implies that $H$ is coamenable in $N$ (see [8] or [22, Theorem 4.18]).

This completes the proof of Theorem 3.3, except for the last statement. To see that it holds, assume that the Furstenberg-Poisson boundary of the $\mu$-random walk on $G / K$ is almost surely trivial. We now invoke Bowen's definition of the Poisson bundle $\mathrm{B}_{\mu}(\lambda)$ as a bundle over $\left(\operatorname{Sub}_{G}, \lambda\right)$, where the fiber over $H<G$ is the Furstenberg-Poisson boundary of the $\mu$-random walk on $H \backslash G$ (see [4], the discussion in the penultimate paragraph of Section 3.3 , as well as (4.3) and the preceding discussion). Since these fibers are all trivial, it follows that $\mathrm{B}_{\mu}(\lambda)$ is isomorphic, as a $G$-space, to $\left(\mathrm{Sub}_{G}, \lambda\right)$. In particular the $G$-action on the $\mathrm{B}_{\mu}(\lambda)$ is measure preserving. Hence, by the argument above, $K$ is co-amenable in $G$.

\section{INTERMEDIATE FACTOR THEOREMS}

Our main results, Theorems 1 and 2, are consequences of Theorem 4.5 below, which is a statement regarding any IRS that satisfies an intermediate factor theorem (IFT). The original proofs of StuckZimmer and of Bader-Shalom are also each based on a corresponding IFT. We start this section by defining intermediate factors.

Let $\Pi(G, \mu)$ be the Furstenberg-Poisson boundary of a group $G$ with a Haar-equivalent probability measure $\mu$, and let $G \curvearrowright(X, m)$ be a 
pmp action. A $G$-quasi-invariant probability space $(Y, \eta)$ is a $(G, \mu)$ intermediate factor over $(X, m)$ if there exist $G$-factors $\kappa$ and $\pi$

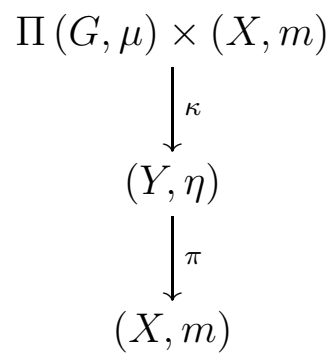

such that the composition $\pi \circ \kappa$ is the natural projection $(p, x) \mapsto x$. Note that, if we denote by $\nu$ the measure of the Furstenberg-Poisson boundary $\Pi(G, \mu)$, then $\kappa_{*}(\nu \times m)=\eta . \pi_{*} \eta=m$ and $[\pi \circ \kappa]_{*}(\nu \times m)=$ $m$.

A trivial example of an intermediate factor is

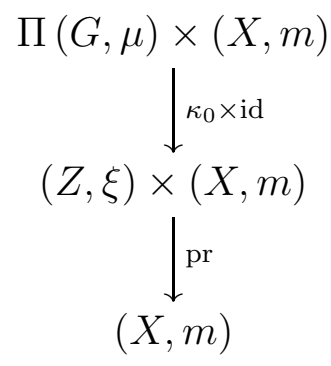

where $(Z, \xi)$ is any $G$-factor of the Furstenberg-Poisson boundary. $G$ factors of the Furstenberg-Poisson boundary $\Pi(G, \mu)$ are also called $(G, \mu)$-boundaries, or $\mu$-proximal actions.

Zimmer [27] proves an intermediate factor theorem, which was generalized (and had its proof corrected) by Nevo and Zimmer [20]. It provides conditions on $G$ and $(X, m)$ under which every intermediate factor is isomorphic to a product $(Z, \xi) \times(X, m)$. Bader and Shalom [3] prove the same result for intermediate factors over irreducible actions of product groups. To state these theorems we first define an IFT action.

Definition 4.1. A pmp $G$-action $G \curvearrowright(X, m)$ is an IFT action if there exists a Haar-equivalent $\mu$ such that for every $(G, \mu)$-intermediate 
factor

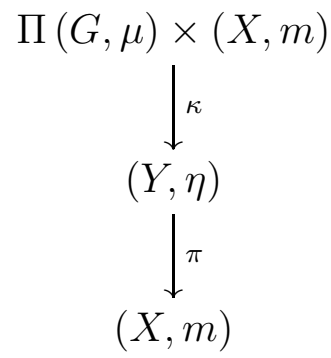

there exists a $G$-factor $(Z, \xi)$ of the Furstenberg-Poisson boundary and $G$-isomorphism $\varphi:(Y, \eta) \rightarrow(Z, \xi) \times(X, m)$ such that the following diagram commutes.

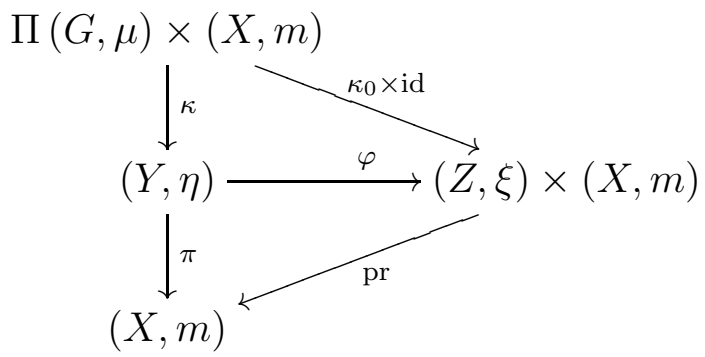

Theorem 4.2 (Intermediate Factor Theorem [3, 27]). Let $G$ be either (1) a product of lcsc groups, or (2) a connected semi-simple Lie group with finite center, no compact factors and higher rank. Then every irreducible pmp G-space is an IFT action.

Recently, Levit [16] proved an intermediate factor theorem over local fields.

4.1. Poisson bundles as intermediate factors. The next claim shows that every Poisson bundle $\mathrm{B}_{\mu}(\lambda)$ is an intermediate factor. This will allow us to apply intermediate factor theorems to Poisson bundles.

Proposition 4.3. Let $\lambda \in \operatorname{IRS}(G)$. Then $\mathrm{B}_{\mu}(\lambda)$ is an intermediate factor. Namely, there exist $G$-maps

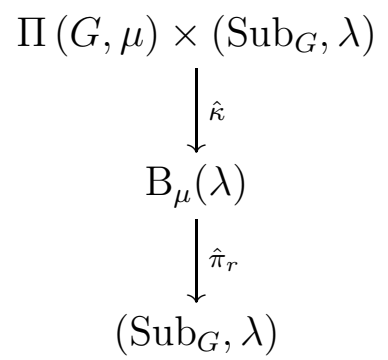


such that $\hat{\pi}_{r} \circ \hat{\kappa}$ is the projection $(b, H) \mapsto H$.

Proof. Recall that $\pi_{r}: \operatorname{Cos}_{G} \rightarrow \operatorname{Sub}_{G}$ is given by $\pi_{r}(H g)=H$, and that it commutes with the left $G$-action: $\pi_{r}(k H g)=\pi_{r}(H g)^{k}$. We extend its definition to a $G$-map $\pi_{r}: \operatorname{Cos}_{G}^{\mathbb{N}} \rightarrow \operatorname{Sub}_{G}$ by

$$
\pi_{r}\left(c_{1}, c_{2}, \ldots\right)=\pi_{r}\left(c_{1}\right) .
$$

Recall that $\kappa: G^{\mathbb{N}} \times \operatorname{Sub}_{G} \rightarrow \operatorname{Cos}_{G}^{\mathbb{N}}$ is given by $\kappa\left(\left(g_{1}, g_{2}, \ldots\right), H\right)=$ $\left(H g_{1}, H g_{2}, \ldots\right)$. Hence the composition $\pi_{r} \circ \kappa$ (see (4.2) below) is equal to the projection $\left(\left(g_{1}, g_{2}, \ldots\right), H\right) \mapsto H$.

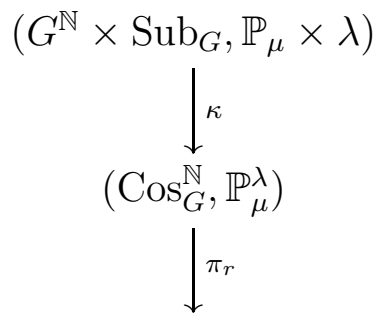

$\left(\mathrm{Sub}_{G}, \lambda\right)$

Define the shift-action on $G^{\mathbb{N}} \times \mathrm{Sub}_{G}$ in the obvious way. Then the shift commutes with $\kappa$, and so the shift-invariant sigma-algebra of $\left(\operatorname{Cos}_{G}^{\mathbb{N}}, \mathbb{P}_{\mu}^{\lambda}\right)$ is a sub-sigma-algebra of the shift-invariant sigma-algebra of $\left(G^{\mathbb{N}} \times \operatorname{Sub}_{G}, \mathbb{P}_{\mu} \times \lambda\right)$. Hence $\kappa$, as a factor between the two shift-invariant sigma-algebras, extends to a factor $\hat{\kappa}$ between the Mackey realizations of these two sigma-algebras, which are, by definition, $\Pi(G, \mu) \times$ $\left(\mathrm{Sub}_{G}, \lambda\right)$ and $\mathrm{B}_{\mu}(\lambda)$.

As we note above, $\mathbb{P}_{\mu}^{\lambda}$ is supported on elements of the form $\left(\mathrm{Hg}_{1}, \mathrm{Hg}_{2}, \ldots\right)$. Since $\pi_{r}\left(H g_{1}, H g_{2}, \ldots\right)=H, \pi_{r}$ is in fact measurable in the shiftinvariant sigma-algebra of $\left(\operatorname{Cos}_{G}^{\mathbb{N}}, \mathbb{P}_{\mu}^{\lambda}\right)$. It follows that, as with $\kappa$ above, $\pi_{r}$ can be extended to a $G$-map $\hat{\pi}_{r}$ of the corresponding Mackey realizations; namely to a $G$-map from $\mathrm{B}_{\mu}(\lambda)$ to $\left(\mathrm{Sub}_{G}, \lambda\right)$. Thus $\hat{\kappa}$ and $\hat{\pi}_{r}$ act as in (4.1).

Finally, since $\pi_{r} \circ \kappa$ is the projection on the second coordinate, then so is $\hat{\pi}_{r} \circ \hat{\kappa}$, and we have proved the claim.

Having shown that every Poisson bundle is an intermediate factor, we explore the consequences of the application of an intermediate factor theorem to one. In particular, when $G \curvearrowright\left(\mathrm{Sub}_{G}, \lambda\right)$ is an IFT action, $\mathrm{B}_{\mu}(\lambda)$ is isomorphic to the product $\left(\operatorname{Sub}_{G}, \lambda\right) \times(Z, \xi)$, where $(Z, \xi)$ is some $(G, \mu)$-boundary. As the next proposition shows, this means that $(Z, \xi)$ is invariant to every element of the normal closure of $\lambda$. 
Proposition 4.4. Let $\lambda \in \operatorname{IRS}(G)$ be such that $G \curvearrowright\left(\mathrm{Sub}_{G}, \lambda\right)$ is an IFT action. Then $\mathrm{B}_{\mu}(\lambda)=(Z, \xi) \times\left(\operatorname{Sub}_{G}, \lambda\right)$, where $(Z, \xi)$ is $\overline{\langle\lambda\rangle}$ invariant.

Before proving this proposition we will need to introduce some additional notation. A natural decomposition of the Poisson bundle $\mathrm{B}_{\mu}(\lambda)$ is by disintegration according to the factor $\hat{\pi}_{r}: \mathrm{B}_{\mu}(\lambda) \rightarrow\left(\mathrm{Sub}_{G}, \lambda\right)$ defined in the proof of Proposition 4.3 above. We denote the fiber $\hat{\pi}_{r}^{-1}(H)$ by $\left(B_{H}, \nu_{H}\right)$; this space can be shown to be the Furstenberg-Poisson boundary of the $\mu$-random walk on $H \backslash G$ [4]. Hence we can write

$$
\mathrm{B}_{\mu}(\lambda)=\left\{(b, H): H \in \operatorname{Sub}_{G}, b \in B_{H}\right\},
$$

where the measure is not displayed explicitly.

Proof of Proposition 4.4. By Proposition 4.3, $\mathrm{B}_{\mu}(\lambda)$ is indeed an intermediate factor. Hence there exists a $G$-isomorphism $\varphi: \mathrm{B}_{\mu}(\lambda) \rightarrow$ $(Z, \xi) \times\left(\operatorname{Sub}_{G}, \lambda\right)$, where $(Z, \xi)$ is a $(G, \mu)$-boundary, and such that the following diagram commutes.

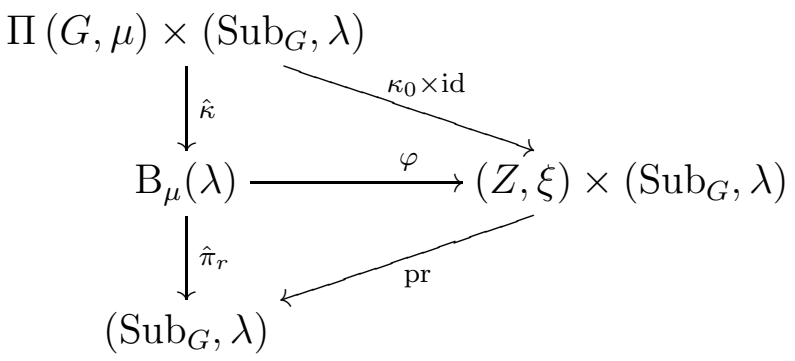

It follows that $\left(B_{H}, \nu_{H}\right)$ (the $\hat{\pi}_{r}$-fiber above $\left.H\right)$ is isomorphic to $(Z, \xi)$ (the pr-fiber above $H$ ), for $\lambda$-almost every $H \in \mathrm{Sub}_{G}$.

Note that for $h \in H$,

$$
\begin{aligned}
h \kappa\left(\left(g_{1}, g_{2}, \ldots\right), H\right) & =h\left(H g_{1}, H g_{2}, \ldots\right) \\
& =\left(H^{h} h g_{1}, H^{h} h g_{2}, \ldots\right) \\
& =\kappa\left(\left(g_{1}, g_{2}, \ldots\right), H\right) .
\end{aligned}
$$

We extend $\kappa$ (as in the proof of Proposition 4.3) but this time to a factor $\tilde{\kappa}$ from $G^{\mathbb{N}} \times \operatorname{Sub}_{G}$ to $\mathrm{B}_{\mu}(\lambda)$. Then $\tilde{\kappa}\left(\left(g_{1}, g_{2}, \ldots\right), H\right)=(b, H)$ for some $b \in B_{H}$. This follows from the fact that $\hat{\pi}_{r}(b, H)=H$, and that $\pi_{r} \circ \kappa$ is the projection. It then further follows from (4.4) that $h(b, H)=(b, H)$, for all $h \in H$; the invariance of $\left(H g_{1}, H g_{2}, \ldots\right)$ to $H$ extends from $\left(\operatorname{Cos}_{G}^{\mathbb{N}}, \mathbb{P}_{\mu}^{\lambda}\right)$ to its shift-invariant sub-sigma-algebra and its Mackey realization.

Since, as we noted above, $\left(B_{H}, \nu_{H}\right)$ is isomorphic to $(Z, \xi)$, it follows that $h(z, H)=(z, H)$ for all $\lambda$-almost every $h \in H$ and $\xi$-almost every 
$z \in Z$. But the $G$-action on $(Z, \xi) \times\left(\operatorname{Sub}_{G}, \lambda\right)$ is the diagonal action, and so we have that $(Z, \xi)$ is $H$-invariant for $\lambda$-almost every $H \in \operatorname{Sub}_{G}$.

By a standard argument 24, we can choose a compact model for $(Z, \xi)$ such that $G \curvearrowright(Z, \xi)$ is continuous, and deduce that $(Z, \xi)$ is $H$ invariant for every $H$ in the support of $\lambda$, and is therefore $\overline{\langle\lambda\rangle}$-invariant.

4.2. IFT actions and co-amenable IRSs. Given the connection between intermediate factors and Poisson bundles, and given Theorem 3.3, we are now ready to show how intermediate factor theorems can be used to show that an IRS is co-amenable.

Theorem 4.5. Let $G$ be an lcsc group. Let $K \leq G$ be an IRS with distribution $\lambda$ such that $G \curvearrowright\left(\mathrm{Sub}_{G}, \lambda\right)$ is an IFT action. Then $K$ is co-amenable in $\overline{\langle\lambda\rangle}$, the normal closure of $\lambda$.

Proof. By Proposition 4.4, if $G \curvearrowright\left(\mathrm{Sub}_{G}, \lambda\right)$ is an IFT action then the action of $\overline{\langle\lambda\rangle}$ on the Poisson bundle $\mathrm{B}_{\mu}(\lambda)$ is measure preserving. Applying Theorem 3.3 yields the desired result.

\section{ProOfs OF MAIN THEOREMS AND COROLLARIES}

Proof of Theorem 11. Let $\lambda \in \operatorname{IRS}(G)$ be the distribution of $K$, and denote $N=\overline{\langle\lambda\rangle}$. By the Bader-Shalom IFT (Theorem 4.2), Theorem 4.5 implies that $K$ is co-amenable in $N$.

Denote by $N_{i} \triangleleft G_{i}$ the closure of the projection of $N$ on $G_{i}$. By Fact 1.1, $N$ is co-abelian in $N_{1} \times N_{2}$, and hence in particular coamenable. As $K$ is co-amenable in $N$, we conclude that $K$ is coamenable in $N_{1} \times N_{2}$.

Proof of Theorem 2. Let $\lambda \in \operatorname{IRS}(G)$ be the distribution of $K$, and denote $N=\overline{\langle\lambda\rangle}$. By the Nevo-Zimmer IFT (Theorem 4.2), Theorem 4.5 implies that $K$ is co-amenable in $N$. Hence if $N=G$ then $K$ is coamenable in $G$, and we are done.

Otherwise $N \neq G$. Then there exists a non-central, closed normal $M \triangleleft G$ such that $N$ and $M$ commute [21]. By the irreducibility of the IRS $M$ acts ergodically on $\left(\mathrm{Sub}_{G}, \lambda\right)$. But since $N$ and $M$ commute, and since $K$ is contained in $N$, this action is trivial. Hence $K$ must equal a closed normal subgroup.

Proof of Corollary 1.2. Let $K$ be an irreducible IRS of $G$. By Theorem 1, there exist subgroups $N_{1} \triangleleft G_{1}$ and $N_{2} \triangleleft G_{2}$ such that $K$ is co-amenable in $N=N_{1} \times N_{2}$. Since $K$ is irreducible, if either $N_{1}$ or $N_{2}$ is trivial then $K$ must equal $N_{1} \times N_{2}$. Otherwise, because $G_{1}$ and $G_{2}$ 
are just non-amenable, $N_{1}$ is co-amenable in $G_{1}$ and $N_{2}$ is co-amenable in $G_{2}$. Hence $N$ is co-amenable in $G$, and so $K$ is co-amenable in $G$.

To prove Corollary [1.5 we will need the following two elementary lemmas. The second one, Lemma [5.2, is a variation on [3, Lemma 4.2] and [23, Lemma 1.8].

Lemma 5.1. Let $H$ be a subgroup of $G_{1} \times G_{2}$. If the projection of $H$ to the second coordinate is equal to $G_{2}$, then the action of $G_{1}$ on $G / H$ is transitive.

Proof. The projection of $H$ to the second coordinate is equal to $G_{2}$; that is, for every $g_{2} \in G_{2}$ there exists an $g_{1} \in G_{1}$ such that $g_{1} g_{2} \in H$. Fix any $g_{1} g_{2} H \in G / H$. Then there exists a $g_{1}^{\prime} \in G_{1}$ such that $g_{1}^{\prime} g_{2}^{-1} \in H$, and

$$
g_{1} g_{1}^{\prime} H=g_{1} g_{1}^{\prime}\left(g_{2} g_{2}^{-1}\right) H=g_{1} g_{2}\left(g_{1}^{\prime} g_{2}^{-1}\right) H=g_{1} g_{2} H,
$$

where the second equality uses the commutativity of $G_{1}$ and $G_{2}$, and the last equality follows from the fact that $g_{1}^{\prime} g_{2}^{-1} \in H$. Hence $G_{1}$ indeed acts transitively on $G / H$.

Lemma 5.2. Let $G=G_{1} \times G_{2}$ be lcsc with $G_{1}$ simple, and let $G \curvearrowright$ $(X, m)$ be a non-trivial, irreducible pmp action. Then the action $G_{1} \curvearrowright$ $(X, m)$ is essentially free.

Proof. Consider the map $s: X \rightarrow \operatorname{Sub}_{G_{1}}$ which assigns to each $x \in X$ its $G_{1}$-stabilizer $s(x)=\left\{g_{1} \in G_{1}: g_{1} x=x\right\}$. This map is easily seen to be $G_{2}$-invariant, by the fact that $G_{1}$ and $G_{2}$ commute. Likewise, it is $G_{1}$-equivariant, and so, by ergodicity, $s(x)$ is $m$-almost surely equal to some closed normal $N_{1} \triangleleft G_{1}$.

Now, if $N_{1}=G_{1}$ then the action $G_{1} \curvearrowright(X, m)$ is trivial, and by irreducibility $G \curvearrowright(X, m)$ is trivial, in contradiction to the claim hypothesis. Otherwise, since $G_{1}$ is simple, $N_{1}$ is trivial, and so the action $G_{1} \curvearrowright(X, m)$ is essentially free.

Proof of Corollary 1.5. Let $G=G_{1} \times G_{2}$, let $G \curvearrowright(X, m)$ be an irreducible pmp action, and let $\lambda$ be the distribution of the IRS $K$ associated with $(X, m)$. By Corollary $1.2, K$ is either equal to a normal subgroup, or $K$ is co-amenable in $G$. In the former case, since $G_{1}$ and $G_{2}$ are simple, $K$ is either trivial, in which case the action is essentially free, or else $K$ is one of $\left\{G_{1}, G_{2}, G\right\}$, and hence by irreducibility the action is trivial. We therefore assume henceforth that $K \neq G$ is coamenable in $G$, and show that this leads to a contradiction, unless the action is trivial or free. 
Let $\mathrm{pr}_{2}: \mathrm{Sub}_{G} \rightarrow \mathrm{Sub}_{G_{2}}$ be the map that assigns to each $H \in \mathrm{Sub}_{G}$ its projection on the second coordinate. Note that $G_{2}$ is discrete, and therefore $\mathrm{pr}_{2} H$ is closed, since every subgroup of $G_{2}$ is closed. This map is easily seen to be $G_{1}$-invariant and $G_{2}$-equivariant, and therefore $\operatorname{pr}_{2} K$ is almost surely equal to some normal $M_{2} \triangleleft G_{2}$, by the ergodicity of the $G_{2}$-action 3 .

Assume first that $M_{2}$ is the trivial subgroup. Then $K$ is contained in $G_{1}$, and so, by the ergodicity of the $G_{2}$-action on $\left(\mathrm{Sub}_{G}, \lambda\right), K$ must almost surely equal some closed normal $M_{1} \triangleleft G_{1}$. As above, if $M_{1}=G_{1}$ then the action is trivial. Otherwise $M_{1}$ is trivial, and we have that $K$ is trivial, so that the action $G \curvearrowright(X, m)$ is essentially free.

Finally, consider the case that $M_{2}$ is not the trivial subgroup. Since $G_{2}$ is simple, $M_{2}=G_{2}$, and the projection of $K$ on the second coordinate is $G_{2}$. Therefore, by Lemma 5.1, $G_{1}$ acts transitively on $G / K$. By Lemma 5.2, $G_{1}$ acts essentially freely on $G / K$. Hence the action $G_{1} \curvearrowright G / K$ is isomorphic to the natural action $G_{1} \curvearrowright G_{1}$. Since $K$ is almost surely co-amenable in $G$, there is a $G$-invariant mean on $G / K$, which in particular is $G_{1}$-invariant. Therefore, and since we identified $G / K$ with $G_{1}$, there exists a $G_{1}$-invariant mean on $G_{1}$. So $G_{1}$ is amenable - contradiction.

In order to prove Corollaries 1.3 and 1.4 we will use (and elaborate on) Varadarajan's ergodic decomposition theorem [24] to show that the existence of invariant probability measures supported on orbits implies that an action is essentially transitive. We state Varadarajan's theorem after the following definition.

Definition 5.3 (Varadarajan [24]). Let $G$ be an lcsc group acting on a standard measurable space $X$. Let $E(X)^{G}$ denote the space of $G$ invariant, ergodic probability measures on $X$. A decomposition map is a measurable map $\beta: X \rightarrow E(X)^{G}$ with the following properties.

(1) $\beta$ is $G$-invariant. I.e., $\beta_{g x}=\beta_{x}$ for all $g \in G$ and $x \in X$.

(2) For every $\eta \in E(X)^{G}$, it holds that $\eta\left(\beta^{-1}(\eta)\right)=1$.

(3) For every $G$-invariant measure $\theta$ it holds that

$$
\theta=\int_{X} \beta_{x} \mathrm{~d} \theta(x)
$$

Theorem 5.4 (Varadarajan [24]). For every action of an lcsc group $G$ on a standard measurable space $X$, there exists a decomposition map $\beta$. Furthermore, $\beta$ is essentially unique, in the sense that if $\beta$ and $\beta^{\prime}$ are decomposition maps then $\theta\left(\left\{x \in X: \beta_{x} \neq \beta_{x}^{\prime}\right\}\right)=0$ for any $G$-invariant probability measure $\theta$.

\footnotetext{
${ }^{3}$ We would like to thank Jesse Peterson for suggesting this argument.
} 
Lemma 5.5. Let $G$ be an lcsc group, and let $G \curvearrowright(X, m)$ be an ergodic pmp action. Assume that there exists a $G$-invariant probability measure on the orbit $G x$ for $m$-almost every $x \in X$. Then the action $G \curvearrowright$ $(X, m)$ is essentially transitive.

Proof. Let $\beta: X \rightarrow E(X)^{G}$ be a decomposition map of $X$ with respect to the $G$-action. Let $x \in X$ be such that there exists a $G$-invariant and ergodic probability measure $\eta_{x}$ with $\eta_{x}(G x)=1$.

By the second property of decomposition maps, there exists an element $y \in G x$ (in fact a $\eta_{x}$-full measure set of such elements) for which $\beta_{y}=\eta_{x}$. Since $\beta$ is $G$-invariant, we get that $\beta_{x}=\eta_{x}$ and in particular $\beta_{x}$ is supported on $G x$.

Let $A$ be an $m$-full measure set of $x \in X$ for which there exists a $G$-invariant, ergodic measure $\eta_{x}$ on $G x$, and for which, by the above, $\beta_{x}=\eta_{x}$ is supported on a $G$-orbit. Then

$$
m=\int_{X} \beta_{x} \mathrm{~d} m(x)=\int_{A} \beta_{x} \mathrm{~d} m(x),
$$

and it follows by the ergodicity of $m$ that it is equal to some $\beta_{x}$. Hence $m$ is supported on a $G$-orbit, or, equivalently, the action is essentially transitive.

The following is a corollary of Lemma 5.5. Recall that $H$ is co-finite in $G$ if there exists a finite, $G$-invariant measure on $G / H$.

Corollary 5.6. Let $G$ be an lcsc group, and let an ergodic IRS $K$ in $G$ be co-finite. Then its distribution is supported on a single orbit $\left\{H^{g}: g \in G\right\}$.

In Stuck-Zimmer this is proven for the case of Lie groups [23, Corollary 3.2].

We next prove an analogue of Lemma 5.5 for almost direct products.

Definition 5.7. Let $G_{1}$ and $G_{2}$ be closed subgroups of $G$. Then $G$ is said to be an almost direct product of $G_{1}$ and $G_{2}$ if the two groups commute and if $G=G_{1} G_{2}$.

Lemma 5.8. Let $G=G_{1} G_{2}$ be an lcsc almost direct product, and let $G \curvearrowright(X, m)$ be a pmp action on a standard measurable space that is $G_{1}$ ergodic. Assume that there exists a $G_{1}$-invariant probability measure on $m$-almost every $G$-orbit. Then the action $G \curvearrowright(X, m)$ is essentially transitive.

Proof. Let $\beta^{1}: X \rightarrow E(X)^{G_{1}}$ be a decomposition map of the $G_{1^{-}}$ action on $X$. Note that, since $G_{1}$ and $G_{2}$ commute, it holds for every 
$\eta \in E(X)^{G_{1}}$ and $g \in G$ that $g \eta \in E(X)^{G_{1}}$, and so $G$ acts on $E(X)^{G_{1}}$. Hence one can consider the question of whether $\beta^{1}$ commutes with $G$.

In fact, in order to follow the same arguments of Lemma 5.5 we will require that $\beta^{1}$ be essentially $G$-equivariant. That is, that there exists an $A \subseteq X$ with $\theta(A)=1$ for any $G_{1}$-invariant measure $\theta$, and such that $g \beta_{x}^{1}=\beta_{g x}^{1}$ for all $g \in G$ and $x \in A$. Assume first that $\beta^{1}$ satisfies this condition.

Since $m$ is $G_{1}$-invariant, $m(A)=1$. Hence there exists an $m$-full measure set $A^{\prime} \subseteq A$ such that there exists a $G_{1}$-invariant and ergodic measure $\eta_{x}$ on $G x$ for all $x \in A^{\prime}$. Fix some $x \in A^{\prime}$. Since $\eta_{x}(G x)=1$, we can find some $y=g x \in G x$ such that $\beta_{y}^{1}=\eta_{x}$. Then

$$
g \beta_{x}^{1}=\beta_{g x}^{1}=\beta_{y}^{1}=\eta_{x}
$$

and so we conclude that $\beta_{x}^{1}$ is supported on $G x$ for every $x \in A^{\prime}$.

Since $m$ is $G_{1}$-invariant we can write

$$
m=\int_{X} \beta_{x}^{1} \mathrm{~d} m(x)=\int_{A^{\prime}} \beta_{x}^{1} \mathrm{~d} m(x)
$$

and, by the $G_{1^{-}}$-ergodicity of $m, m=\beta_{x}^{1}$ for some $x \in A^{\prime}$ and in particular $m(G x)=1$. Hence the action $G \curvearrowright(X, m)$ is essentially transitive.

Finally, we argue that $\beta^{1}$ is essentially $G$-equivariant. Following Varadarajan, let $\mathcal{F}$ be the Banach space of all bounded measurable functions on $X$. Let $U^{1}: \mathcal{F} \rightarrow \mathcal{F}$ be the operator defined by $U^{1}(f)(x)=$ $\int_{X} f(y) \mathrm{d} \beta_{x}^{1}(y)$, for any $f \in \mathcal{F}$. Note that by definition, the equivariance of $\beta^{1}$ is equivalent to the equivariance of $U^{1}$.

Now, for any $G_{1}$-invariant measure $\theta, U^{1}$ is the conditional expectation defined by the factor $(X, \theta) \rightarrow G_{1} \backslash(X, \theta)$, the space of $G_{1}$-ergodic components of $(X, \theta)$ :

$$
U^{1}: L^{\infty}(X, \theta) \rightarrow L^{\infty}\left(G_{1} \backslash(X, \theta)\right) .
$$

Since the actions of $G_{1}$ and $G_{2}$ on $X$ commute, $U^{1}$, as the conditional expectation map, is $G_{2}$-equivariant. Hence $U^{1}: \mathcal{F} \rightarrow \mathcal{F}$ is $G$-equivariant on a $\theta$-full measure set. Since this holds for any $G_{1^{-}}$ invariant measure $\theta$, we conclude that $U^{1}$, and so the associated decomposition map $\beta^{1}$, are essentially $G$-equivariant, and the proof is complete.

As a final lemma before the proofs of Corollaries 1.3 and 1.4 we note the following fact regarding property $(\mathrm{T})$ groups. It is a straightforward generalization of the fact that any property $(\mathrm{T})$ group that is also amenable must admit a finite invariant measure. 
Claim 5.9. Let $G$ be an lcsc group, let $H \in \mathrm{Sub}_{G}$ be co-amenable. Let $G^{\prime} \in \mathrm{Sub}_{G}$ be any closed subgroup with property $(T)$. Then there exists a $G^{\prime}$-invariant probability measure on $G / H$. In particular, if $G$ has property $(T)$ then $H$ is co-finite in $G$.

Proof. Since $H$ is co-amenable in $G$, the quasi-regular representation of $G$ on $L^{2}(G / H)$ weakly contains the trivial representation; equivalently, there are almost-invariant non-zero vectors in this representation (see [22, Theorem 4.18 and Definition 5.1]).

Restrict this representation to a representation of $G^{\prime}$. Then this restricted representation also has almost-invariant non-zero vectors. Since $G^{\prime}$ has property (T) this implies that there also exist $G^{\prime}$-invariant unit vectors.

A unit $G^{\prime}$-invariant vector in this representation corresponds to a function $f \in L^{2}(G / H, \nu)$, for some $G$-quasi-invariant measure $\nu$, such that for all $g \in G^{\prime}$ and $\nu$-almost every $x \in G / H$

$$
f(x)=f\left(g^{-1} x\right) \sqrt{\frac{\mathrm{d} g_{*} \nu}{\mathrm{d} \nu}(x)},
$$

and such that $\int|f|^{2} d \nu=1$.

Define $\nu^{\prime}$ by $\mathrm{d} \nu^{\prime}(x)=|f(x)|^{2} \mathrm{~d} \nu(x)$. Then for any $g \in G^{\prime}$

$$
\begin{aligned}
\mathrm{d}\left(g_{*} \nu^{\prime}\right) & =\left|f\left(g^{-1} x\right)\right|^{2} \mathrm{~d} \nu\left(g^{-1} x\right) \\
& =|f(x)|^{2} \frac{\mathrm{d} \nu}{\mathrm{d} g_{*} \nu}(x) \mathrm{d}\left(g_{*} \nu\right)(x) \\
& =|f(x)|^{2} \mathrm{~d} \nu(x) \\
& =\mathrm{d} \nu^{\prime} .
\end{aligned}
$$

Hence $\nu^{\prime}$ is $G^{\prime}$-invariant. Finally,

$$
\nu^{\prime}(G / H)=\int_{G / H}|f(x)|^{2} \mathrm{~d} \nu(x)=1,
$$

and so $\nu^{\prime}$ is a probability measure.

We are now ready to prove Corollaries 1.3 and 1.4 .

Proof of Corollary 1.3. Let $K$ be the associated stabilizer IRS. By Theorem 1, there exists a normal subgroup $N=N_{1} \times N_{2} \triangleleft G$ such that $K$ is co-amenable in $N$.

Since $G_{1}$ is just non-amenable, either $N_{1}$ is co-amenable in $G_{1}$ or else $N_{1}$ is trivial. In the latter case we have that $K$ is contained (in fact, co-amenable) in $\{e\} \times N_{2}$, and since $K$ is irreducible, it must almost surely equal $\{e\} \times N_{2}^{\prime}$, for some $N_{2}^{\prime} \triangleleft G_{2}$. Since the action $G \curvearrowright(X, m)$ is faithful, $N_{2}^{\prime}=\{e\}$ and the action is essentially free. 
We are therefore left with the case that $N_{1}$ is co-amenable in $G_{1}$. Then since $K$ is co-amenable in $N_{1} \times N_{2}$ we get that $K$ is co-amenable in $G_{1} \times N_{2}$. It follows that, since $G_{1}$ has property $(\mathrm{T}), m$-almost every $G_{1} \times N_{2}$-orbit admits a $G_{1}$-invariant probability measure (Claim 5.9), and so each $G$-orbit admits a $G_{1}$-invariant probability measure. It follow from Lemma 5.8 that $G \curvearrowright(X, m)$ is essentially transitive, and hence $K$ is co-finite.

Proof of Corollary 1.4. Assume that $G_{1}$ is a simple factor of $G$ with property $(\mathrm{T})$, and note that by Theorem $2 K$ is either almost surely equal to a normal subgroup $N$, or $K$ is co-amenable in $G$.

In the former case, since the action is faithful, the only possibility is $N=\{e\}$, and then the action is essentially free.

In the latter case, the same argument of the proof of Corollary 1.3 above shows that $G \curvearrowright(X, m)$ is essentially transitive and $K$ is co-finite in $G$.

Finally, it follows from the Borel Density Theorem that $K$ is discrete, and hence a lattice in $G$.

\section{REFERENCES}

[1] M. Abert, N. Bergeron, I. Biringer, T. Gelander, N. Nikolov, J. Raimbault, and I. Samet, On the growth of $L^{2}$-invariants for sequences of lattices in Lie groups, arXiv preprint arXiv:1210.2961 (2012).

[2] M. Abért, Y. Glasner, and B. Virág, Kestens theorem for invariant random subgroups, Duke Mathematical Journal 163 (2014), no. 3, 465-488.

[3] U. Bader and Y. Shalom, Factor and normal subgroup theorems for lattices in products of groups, Inventiones Mathematicae 163 (2006), no. 2, 415-454.

[4] L. Bowen, Random walks on random coset spaces with applications to furstenberg entropy, Inventiones Mathematicae 196 (2014), no. 2, 485-510.

[5] C. Chabauty, Limite d'ensembles et géométrie des nombres, Bulletin de la Société Mathématique de France 78 (1950), 143-151.

[6] D. Creutz, Stabilizers of actions of lattices in products of groups, arXiv preprint arXiv:1305.3648 (2013).

[7] D. Creutz and J. Peterson, Stabilizers of ergodic actions of lattices and commensurators, arXiv preprint arXiv:1303.3949 (2013).

[8] P. Eymard, Moyennes invariantes et representations unitaires, Lecture Notes in Mathematics, Springer-Verlag, 1972.

[9] H. Furstenberg, Random walks and discrete subgroups of Lie groups, Advances in Probability and Related Topics 1 (1971), 1-63.

[10] _ Boundary theory and stochastic processes on homogeneous spaces, Proceedings of symposia in pure mathematics, 1974, pp. 193-229.

[11] Y. Hartman and O. Tamuz, Furstenberg entropy realizations for virtually free groups and lamplighter groups, Journal d'Analyse Mathématique 126 (2015), no. $1,227-257$. 
[12] V.A. Kaimanovich, Measure-theoretic boundaries of Markov chains, 0-2 laws and entropy, Proceedings of the conference on harmonic analysis and discrete potential theory, 1992, pp. 145-180.

[13] _ The Poisson boundary of amenable extensions, Monatshefte für Mathematik 136 (2002), no. 1, 9-15.

[14] _ Amenability and the Liouville property, Israel Journal of Mathematics 149 (2005), no. 1, 45-85.

[15] V.A. Kaimanovich and A.M. Vershik, Random walks on discrete groups: boundary and entropy, Ann. Probab. 11 (1983), no. 3, 457-490.

[16] A. Levit, The Nevo-Zimmer intermediate factor theorem over local fields, arXiv preprint arXiv:1404.7007 (2014).

[17] G.W. Mackey, Induced representations of locally compact groups $i$, Annals of Mathematics (1952), 101-139.

[18] — Point realizations of transformation groups, Illinois Journal of Mathematics 6 (1962), no. 2, 327-335.

[19] N. Monod and S. Popa, On co-amenability for groups and von Neumann algebras, Comptes Rendus Mathématiques de l'Académie des Sciences; La Société Royale du Canada 25 (2003), no. 3, 82-87.

[20] A. Nevo and R.J. Zimmer, A generalization of the intermediate factors theorem, Journal d'Analyse Mathématique 86 (2002), no. 1, 93-104.

[21] D.L. Ragozin, A normal subgroup of a semisimple Lie group is closed, Proceedings of the american mathematical society, 1972, pp. 632-633.

[22] Y. Shalom, Invariant measures for algebraic actions, Zariski dense subgroups and Kazhdans property (T), Transactions of the American Mathematical Society 351 (1999), no. 8, 3387-3412.

[23] G. Stuck and R.J. Zimmer, Stabilizers for ergodic actions of higher rank semisimple groups, Annals of Mathematics (1994), 723-747.

[24] V.S. Varadarajan, Groups of automorphisms of Borel spaces, Transactions of the American Mathematical Society 109 (1963), no. 2, 191-220.

[25] F. Wattenberg, Topologies on the set of closed subsets., Pacific Journal of Mathematics 68 (1977), no. 2, 537-551.

[26] R.J. Zimmer, Amenable ergodic group actions and an application to Poisson boundaries of random walks, Journal of Functional Analysis 27 (1978), no. 3, $350-372$.

[27] _ Ergodic theory, semisimple Lie groups, and foliations by manifolds of negative curvature, Publications Mathématiques de l'IHÉS 55 (1982), no. 1, $37-62$.

(Y. Hartman) Weizmann Institute of Science.

(O. Tamuz) California Institute of Technology. 